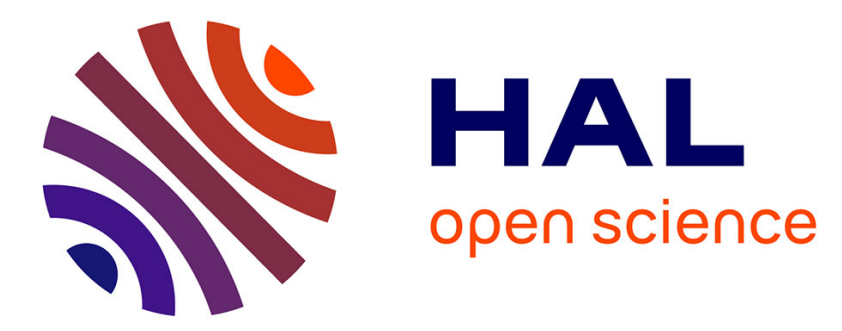

\title{
Cost-effectiveness of preoperative magnetic resonance imaging to optimize surgery in ductal carcinoma in situ of the breast
}

Marguerite Kandel, Ariane Dunant, Corinne S. Balleyguier, Julia Bonastre

\section{To cite this version:}

Marguerite Kandel, Ariane Dunant, Corinne S. Balleyguier, Julia Bonastre. Cost-effectiveness of preoperative magnetic resonance imaging to optimize surgery in ductal carcinoma in situ of the breast. European Journal of Radiology, 2020, 129, 10.1016/j.ejrad.2020.109058 hal-02883161

\section{HAL Id: hal-02883161 https://hal.science/hal-02883161}

Submitted on 8 Jul 2020

HAL is a multi-disciplinary open access archive for the deposit and dissemination of scientific research documents, whether they are published or not. The documents may come from teaching and research institutions in France or abroad, or from public or private research centers.
L'archive ouverte pluridisciplinaire HAL, est destinée au dépôt et à la diffusion de documents scientifiques de niveau recherche, publiés ou non, émanant des établissements d'enseignement et de recherche français ou étrangers, des laboratoires publics ou privés.

\section{(ㅇ)(1) $\$$}

Distributed under a Creative Commons Attribution - NonCommercial - NoDerivatives| 4.0 
Cost-effectiveness of preoperative magnetic resonance imaging to optimize surgery in ductal carcinoma in situ of the breast

Marguerite Kandel, PharmD ${ }^{1,2}$, Ariane Dunant, $\mathrm{PhD}^{1}$, Corinne Balleyguier, MD, $\mathrm{PhD}^{3}$, Julia Bonastre, $\mathrm{PhD}^{1,2}$

${ }^{1}$ Gustave Roussy, Service de Biostatistique et d'Epidémiologie, Villejuif, F-94805, France

${ }^{2}$ CESP, Fac. de médecine - Univ. Paris-Sud, Fac. de médecine - UVSQ, INSERM, Université Paris-Saclay, Villejuif 94805, France

${ }^{3}$ Gustave Roussy, Department of Medical Imaging, Villejuif, F-94805, France

Institution:

Gustave Roussy

114 rue Edouard Vaillant

94805 Villejuif Cedex, France

Corresponding Author:

Corinne BALLEYGUIER, MD, PhD

Gustave Roussy

114 rue Edouard Vaillant 94805 Villejuif Cedex, France

Tel: 33142115094

E-mail: corinne.balleyguier@gustaveroussy.fr 


\section{ABSTRACT \\ PURPOSE}

Complete surgical excision is the main factor for successful breast-conserving surgery in patients with ductal carcinoma in situ (DCIS) of the breast. Preoperative magnetic resonance imaging (MRI) may allow surgery optimization in this indication. From an economic standpoint, systematic preoperative MRI is associated with an extra cost, which may be offset by a decrease in the number of re-interventions. We

performed an economic evaluation alongside IRCIS randomised controlled trial (NCT01112254) to determine whether systematic preoperative MRI in DCIS is a costeffective strategy.

\section{METHODS}

360 patients were included in IRCIS trial. Costs were assessed from the French national health insurance perspective. Resource use was prospectively collected during a 6-month period after randomisation. We estimated the mean cost per averted re-intervention.

\section{RESULTS}

Despite extra costs due to MRI and additional biopsies, difference in total costs between arms was not statistically significant (mean cost of $€ 9,980$ in MRI arm and $€ 9,682$ in no MRI arm, cost difference: $€ 298$ [Cl$\left.\left.\left.\right|_{95 \%}:-470 ; 1,063\right]\right)$. There was a nonsignificant decrease in the rate of re-hospitalisations for positive or close margins (20\% in MRI arm versus $27 \%$ in No MRI arm, difference $\left.-7 \%\left[\mathrm{Cl}_{95 \%}:-17 ; 3\right]\right)$. At a willingness to pay of $€ 500$ to avert a re-intervention, the probability that MRI strategy is cost-effective was $93 \%$. 


\section{CONCLUSION}

Systematic preoperative MRI in patients with DCIS of the breast may be a costeffective strategy. However, the modest clinical benefit associated with such a strategy limits the interest for this procedure in routine practice given the current MRI techniques.

Keywords: Magnetic resonance imaging, Ductal carcinoma in situ of the breast, Cost-effectiveness, Re-intervention 


\section{INTRODUCTION}

Ductal carcinoma in situ (DCIS) of the breast represents approximately $15 \%$ of newly diagnosed breast cancer cases in France ${ }^{1}$. Non-extensive DCIS are treated by breast-conserving surgery followed by radiotherapy. A complete removal of the tumor is the main factor of success for this conservative surgical treatment. However, complete surgical excision is not always possible at the outset due to suboptimal preoperative and per-operative evaluation of the extent of the lesions by standard imaging (mammography, ultrasound). As a consequence, re-intervention for positive or close margins is often required in DCIS, with re-intervention rates ranging from 17 to $58 \%{ }^{2}$.

Three randomised controlled trials (RCTs) $)^{3,4}, 5$ assessed the impact of preoperative magnetic resonance imaging (MRI) of the breast on short-term surgical outcomes. In the COMICE trial ( $n=1,623$ breast cancer patients) conducted in the United Kingdom, the rate of re-intervention for positive or close margins was not significantly lower in the MRI arm (153/816 (19\%) versus 156/807 (19\%), $p=0.77)^{3}$. The MONET trial conducted in the Netherlands assessed the rate of additional surgical procedures (reexcisions and conversion to mastectomy) in patients with a non-palpable breast cancer. Paradoxically, the rate of re-intervention for positive or close margins was higher in the MRI arm (18/53 (34\%) versus 6/50 (12\%), $p=0.008)$. The POMB trial enrolled 440 patients with breast cancer younger than 56 years in three Swedish hospitals. The vast majority of the patients in the POMB study had invasive breast cancer. The breast re-intervention rate was lower in the MRI arm (11/220 (5\%) versus $33 / 220(15 \%), p<0.001)^{5}$. These three RCTs included only a low proportion of DCIS patients (6\% in the COMICE trial and $18 \%$ in the MONET trial), in whom the benefit of preoperative MRI in DCIS is likely to be higher. The proportion of patients 
with DCIS in the POMB study was not reported but was likely to be very low as this study was not selected in a meta-analysis examining the impact of preoperative MRI on the surgical outcomes in patients with $\mathrm{DCIS}^{6}$.

The multicentric IRCIS trial focused on patients with DCIS. It aimed to determine whether the addition of MRI to standard radiological evaluation may improve the assessment of tumor extension and reduce the re-intervention rate for positive or close margins in patients with DCIS undergoing breast-conserving surgery. The results of the IRCIS trial have been published elsewhere ${ }^{7}$. In brief, the re-intervention rate for close or positive margins was $20 \%$ in MRI arm and $27 \%$ in control arm, i.e. a relative reduction of $26 \%$ (stratified OR $0.68,\left[\mathrm{Cl}_{95 \%}: 0.41,1.1\right], \mathrm{p}=0.13$ in the intention to treat analysis and stratified OR $0.59,\left[\mathrm{Cl}_{95 \%}: 0.35,1\right], \mathrm{p}=0.05$ in the per protocol analysis).

From an economic standpoint, systematic preoperative MRI in DCIS patients is associated with an extra cost. However, a potential decrease in re-interventions for positive or close margins and related costs may offset this extra cost. No economic evidence is currently available in DCIS patients and it was a secondary objective of the IRCIS trial to tackle this issue. Our objective was to perform an economic evaluation alongside the IRCIS trial, using patient level data, to determine whether preoperative MRI in DCIS patients is a cost-effective strategy. 


\section{MATERIALS AND METHODS}

\section{IRCIS trial design}

The IRCIS study ${ }^{7}$ (NCT01112254) is a randomised, multicenter phase III trial comparing the use of preoperative MRI (MRI arm) versus standard radiologic evaluation without MRI (No MRI arm) in DCIS accessible to breast-conserving surgery. Ten centers participated in the trial including 8 cancer centers and 2 teaching hospitals. The primary outcome measure was the rate of intervention for positive or close margins in the 6 months following the randomisation. The decision of re-intervention was made according to the histological analysis of the resection margins. Re-intervention (resumption of the tumor bed or mastectomy) was suggested if there were lateral positive or close margins of less than $2 \mathrm{~mm}^{8}$, except for anatomical limits (skin or muscle).

\section{Resource use and costs}

The economic evaluation was conducted from the perspective of the French National Health Insurance. Healthcare resource use was prospectively collected during a 6month period after the randomisation and included all breast cancer-related hospitalisations, visits to the specialists, all imaging procedures and biopsies, radiotherapy, transportations and sick leaves. Transportation for surgery and sick leave costs were collected from the patients using a self-administered questionnaire.

The unit cost data are provided in Table 1 . The tariffs per diagnosis-related groups (DRG) were used to cost each hospital stay and radiotherapy. Reimbursement tariffs from the French national health insurance were used for all procedures (including MRIs and biopsies) and visits to the specialists. No reimbursement tariff was available for MRI-guided macrobiopsy. A micro-costing study was carried out to assess the cost of this procedure from the hospital point of view. The cost of a sick 
leave was computed using its length and the mean daily compensation paid by the National Health Insurance ${ }^{9}$. As regards transportation costs, we calculated the distance between the patient's home (zip code of residence) and the hospital. Distances for trips back and forth were valued using the tariffs from the National Health Insurance per type of transportation. We used descriptive statistics for both resource use and costs and computed 95\% confidence intervals of mean cost difference between arms. All costs are expressed in Euros 2017. Costs were not discounted as the time horizon was 6 months ${ }^{10}$.

\section{Cost-effectiveness analysis}

Economic analyses where costs and outcomes are considered separately ignoring their joint distribution lead to incorrect inference regarding cost-effectiveness ${ }^{11}$. Costeffectiveness analysis (CEA) represents joint uncertainty associated with costs and outcomes. Results of CEAs are expressed as the difference in costs divided by the differences in health outcomes between two strategies: the incremental costeffectiveness ratio (ICER). In our study, the ICER was expressed as the incremental cost per averted re-intervention. It was calculated as the cost difference between arms divided by the difference in the re-intervention rates. To address uncertainty surrounding costs and effectiveness, ninety-five percent confidence intervals for difference in costs, difference in effectiveness and a cost-effectiveness acceptability curve were computed using 10,000 non-parametric bootstrap replications (percentile method). The bootstrap method is a resampling technique used to estimate statistics on a population by sampling a dataset with replacement. A cost-effectiveness acceptability curve (which was estimated from the bootstrap replicates) represents the probability that preoperative MRI is cost-effective compared to standard imaging for various willingness-to-pay thresholds to avert a re-intervention. Missing data for 
sick leaves (patients under 65 years of age) and transportation costs were imputed with the mean. To test the robustness of our results, we performed a sensitivity analysis (Data Supplement). First, we performed a one-way sensitivity analysis varying the unit cost of $M R I$, as the unit cost of $M R I$ reimbursed by the French national health insurance is dependent on the equipment characteristics (date of acquisition and volume of activity). Second, we removed sick leaves and transportation costs because of important missing data for these resources. Finally, a sensitivity analysis excluding the cost of re-intervention was performed. The checklist items from the Consolidated Health Economic Evaluation Reporting Standards were used to report this cost-effectiveness study ${ }^{12,13}$. Statistical analysis was performed with SAS software (version 9.4; SAS Institute, Cary, North Carolina) version 9.4. 


\section{RESULTS}

\section{Patient population}

Three hundred and sixty patients were randomised in the IRCIS trial. Eight patients were excluded for major protocol deviation, 1 patient died from another cause than breast cancer before the assessment of the primary endpoint, 2 patients withdrew their consent, and information for primary endpoint and resource use was missing for 4 patients. In total, 15 out of 360 patients were excluded from the economic evaluation (6\%). Our study population consisted of 345 patients (173 patients randomised in the MRI arm and 172 patients in the No MRI arm (Figure S1)).

\section{Cost analysis}

The healthcare resource use for breast cancer treatment over the 6-month period after randomisation is presented in Table 2. The number of re-hospitalisations related to breast cancer was 48 in the MRI arm and 59 in the No MRI arm, with an average of re-hospitalisations per patient of 0.28 in the MRI arm and 0.34 in the No MRI arm. In the MRI arm, patients had fewer re-interventions for positive or close margins than in the No MRI arm: $20 \%$ in the MRI arm (35/173) and $27 \%$ in the control arm (47/172), although the difference was statistically non-significant (difference of $-7 \%$ $\left[\mathrm{Cl}_{95 \%}:-17 \%\right.$ to $\left.3 \%\right]$ ) in the intention to treat analysis (Table 3).

Overall, mean cost of DCIS management during the 6 months after randomisation amounted to $€ 9,682$ in the No MRI arm and to $€ 9,980$ in the MRI arm (Table 4). The cost difference was $\left.€ 298\left[\mathrm{Cl}_{95 \%}:-470 ; 1,063\right]\right)$. The imaging procedures were the only cost component where difference was statistically significant: the cost of procedures was higher in the MRI arm ( $€ 545$ versus $€ 247$, cost difference $€ 298$ [Cl $\left.{ }_{95 \%}: 237 ; 358\right]$ ) because patients had an additional MRI in this arm compared to the No MRI arm. There was no statistical difference for the other cost components, 
as all confidence intervals of cost differences contained 0 (Table 4). Although not significant, there was a decrease in the cost of re-hospitalisations for positive or close margins (€666 versus $€ 931$, difference $€-265\left[\mathrm{Cl}_{95 \%}:-633 ; 100\right]$ ). Major cost drivers were radiotherapy (€3,258 versus $€ 3,271$, cost difference $€ 13\left[\mathrm{Cl}_{95 \%}:-399 ; 427\right]$ ), sick leaves ( $€ 2,554$ versus $€ 2,647$, cost difference $€ 93\left[\mathrm{Cl}_{95 \%}:-525 ; 683\right]$ ) and hospitalisation for initial surgery $\left(€ 2,396\right.$ versus $€ 2,458$, cost difference $€ 62\left[\mathrm{Cl}_{95 \%}\right.$ : 79; 195]).

\section{Cost-effectiveness analysis}

The cost-effectiveness plane is shown on Figure 1. It shows that in $92 \%$ of the cases (bootstrap replicates), the MRI strategy was more effective (less re-interventions in the MRI arm) but is also more costly in $78 \%$ of the cases. The cost-effectiveness acceptability curve (Figure 2) represents the probability that preoperative MRI is costeffective compared to standard imaging for various willingness-to-pay thresholds to avert a re-intervention (up to $€ 2,500$ per averted re-intervention). For a willingness to pay of $€ 500$ to avert a re-intervention, the probability that the MRI strategy is costeffective was $93 \%$ (Figure 2). Results were similar in the sensitivity analysis (Data supplement). The cost analysis excluding sick leaves and transportations, and the cost analysis in which we varied $( \pm 10 \%)$ the cost of $M R I$ equipment led to similar results in terms of cost-effectiveness (Data Supplement). 


\section{DISCUSSION}

The IRCIS study aimed to assess the clinical impact of systematic preoperative MRI in terms of averted re-intervention for close or positive margins in DCIS patients. The study found a non-significant decrease of re-intervention rate in the MRI arm, which was deemed insufficient to be clinically relevant ${ }^{7}$. Our economic evaluation suggested that MRI strategy may however be a cost-effective strategy in the French context. Cost-effectiveness analyses are decision-support tools that provide information on the uncertainty surrounding both the clinical results and the costs. In the IRCIS trial, clinical results were not statistically significant but in favor of the MRI strategy. In the ITT analysis, patients who did not comply with the allocated strategy (with or without preoperative MRI) were kept in their allocated arm for the analysis whereas they were excluded in the per-protocol analysis. The relative reduction of the re-intervention rate was $26 \%(p=0.13)$ in the ITT analysis and $33 \%(p=0.05)$ in the per-protocol analysis. These results suggest that the IRCIS trial may have been underpowered to show a clinically relevant benefit of a systematic preoperative MRI in DCIS patients.

Regarding the difference in cost between strategies, there was an extra cost corresponding to the cost of systematic MRI which was not offset by a decrease in hospital costs which was rather modest due to a limited number of re-interventions for positive or close margins ( 35 hospitalisations in the MRI arm versus 47 in the no MRI arm).There have been only few cost-effectiveness studies in the field of breast cancer MRI and none in the surgical management of DCIS. This may be explained by the fact that the outcome of imaging procedures does not necessarily translate into different therapeutic pathways. In breast cancer, most of the studies assessed the value of MRI in the diagnosis for women at high risk of developing breast cancer ${ }^{14-17}$. 
One study investigated the impact of MRI in the surveillance of women who had previously breast cancer treated with surgery ${ }^{18}$. Three RCTs assessed the addition of preoperative MRI in all types of breast cancer ${ }^{19,20,5}$, but only one study included a cost-effectiveness analysis. The COMICE ${ }^{19}$ trial, an RCT conducted in the United Kingdom, aimed to determine whether the addition of a preoperative MRI decreases the rate of re-intervention in patients with localized or invasive breast cancer. This trial included 1,623 patients recruited in 45 centers. Our cost results were consistent with this trial. Indeed, in the COMICE trial, mean costs per patient were not different between the arms ( $€ 6,249$ versus $€ 5,914)$. Overall costs were lower than in our study because the cost of sick leaves and transportations were not included in the COMICE study. Excluding these cost components in our study led to similar cost estimates to those in the COMICE trial (Data Supplement).

Our study has limitations which have to be mentioned. First, the rate of missing data for sick leaves and transportation questionnaires was high. However, costeffectiveness results were similar when removing this cost component from the analysis (Data Supplement). Second, we chose to conduct a cost-effectiveness analysis rather than a cost-utility analysis. In the COMICE trial, the quality of life of patients was assessed ${ }^{19}$. The EQ-5D score, at 12 months post initial surgery, was respectively 0.810 in the MRI arm and 0.811 in the No MRI arm. These results suggested that there was no difference in terms of quality of life between the two strategies. In the IRCIS trial, only one-third of the women were re-operated in the No $\mathrm{MRI}$ arm and MRI reduced the risk (absolute reduction) of re-intervention by $7 \%$, resulting in 12 re-interventions being averted. Therefore, it is likely that our study would have been underpowered to demonstrate a significant gain in QALYs. The IRCIS trial focused on DCIS, a cancer of good prognosis, in which the aim is to 
improve the quality of care optimizing breast surgery. In such a case, no survival gain is expected and it is likely that the impact on QALYs would be very limited. Third, there is a debate on whether or not one should include the cost of averted events in the computation of the cost of a strategy in a cost-effectiveness analysis. Mullins et $\mathrm{al}^{21}$ underlined that double counting may occur when the benefit of a treatment or a strategy is included in both the estimation of cost difference (numerator) and the estimation of effectiveness (denominator). However, a consensus on this issue has not yet been reached, and, in the literature, cost-effectiveness studies consider the cost of avoided events in the computation of the cost-effectiveness ratio ${ }^{17,18}$. In this article, the main analysis considered the cost of avoided re-interventions. However, cost-effectiveness results were very similar when excluding the cost of reinterventions for positive or close margins (Data Supplement). Finally, costs assessed in this study do not include all costs incurred from DCIS suspicion as well as long-term follow-up costs. In the IRCIS trial, patients were enrolled when a surgical procedure had been decided. Costs included preoperative imaging and biopsies procedures, hospital costs for surgery (including sentinel lymph node biopsies and re-interventions) and follow-up costs during a 6-month time horizon. However, surgery and adjuvant radiation therapy are expected to be the major cost drivers in the management of patients with DCIS.

In conclusion, the addition of preoperative MRI in DCIS may be a cost-effective strategy in France and our results are likely to be transferable to European countries with a similar healthcare system. However, with current imaging techniques, the modest clinical benefit associated with such a strategy limits the interest this procedure in routine practice. 
Funding information: This research study was sponsored by the French National Cancer Institute (PHRC09_02-005)

\section{Acknowledgments}

This research study was sponsored by the French National Cancer Institute (PHRC09_02-005). The authors thank all radiologists, surgeons and clinical research assistants involved in this trial. The authors thank specifically Mrs Gisele Goma, Mr Soufian Houssaimi and Pascal Ohouo for their work in managing data and trial organization. We are grateful to the directors of medical information from the investigative centers of the IRCIS trial who provided diagnoses related groups for all hospitalizations. 


\section{REFERENCES}

1. Lastier D, Salines E, Danzon A: Programme de dépistage du cancer du sein en France : résultats 2007-2008, évolutions depuis 2004. Inst Veill Sanit , 2011

2. Jansen SA: Ductal Carcinoma In Situ: Detection, Diagnosis, and Characterization with Magnetic Resonance Imaging. Semin ultrasound CT MRI 32:306-318, 2011

3. Turnbull L, Brown S, Harvey I, et al: Comparative effectiveness of MRI in breast cancer (COMICE) trial: a randomised controlled trial [Internet]. Lancet 375:563-571, 2010Available from: http://dx.doi.org/10.1016/S0140-6736(09)62070-5

4. Peters NHGM, Borel Rinkes IHM, Mali WPTM, et al: Breast MRI in nonpalpable breast lesions: a randomized trial with diagnostic and therapeutic outcome - MONET - study. [Internet]. Trials 8:40, 2007Available from:

http://www.pubmedcentral.nih.gov/articlerender.fcgi?artid=2222222\&tool=pmcentrez \&rendertype=abstract

5. Gonzalez V, Sandelin K, Karlsson A: Preoperative MRI of the Breast ( POMB ) Influences Primary Treatment in Breast Cancer : A Prospective, Randomized, Multicenter Study. world J Surg 1685-1693, 2014

6. Fancellu A, Turner RM, Dixon JM, et al: Meta-analysis of the effect of preoperative breast MRI on the surgical management of ductal carcinoma in situ. Br J Surg 883893,2015

7. Balleyguier C, Dunant A, Ceugnart $L$, et al: Breast magnetic resonance imaging (MRI) for local ductal carcinoma in situ staging: multicentric randomized controlled trial to assess the efficacy of preoperative MRI to optimize breast surgery (IRCIS 
Trial). J Clin Oncol , 2019

8. Kell MR, Morrow M: An adequate margin of excision in ductal carcinoma in situ. [Internet]. BMJ 331:789-90, 2005Available from:

http://www.pubmedcentral.nih.gov/articlerender.fcgi?artid=1246064\&tool=pmcentrez \&rendertype=abstract

9. Cedex P: Chapitre XVIII Les indemnités journalières versées au titre de la maladie par le régime général, 2012

10. Haute autorité de santé: Choix méthodologiques pour I ' évaluation économique à la HAS, 2011

11. Briggs AH, Brien BJO, Blackhouse G: Thinking outside the box: Recent Advances in the Analysis and Presentation of Uncertainty in Cost-Effectiveness

Studies. Annu Rev Public Health 377-401, 2002

12. Husereau D, Drummond M, Petrou S, et al: Consolidated Health Economic Evaluation Reporting Standards (CHEERS) statement. Eur J Heal Econ 14:367-372, 2013

13. Ramsey S, Willke R, Glick H, et al: Cost-Effectiveness Analysis Alongside Clinical Trials II - An ISPOR Good Research Practives Task Force Report. Value Heal 18:161-172, 2015

14. de Bock GH, Vermeulen KM, Jansen L, et al: Which screening strategy should be offered to women with BRCA1 or BRCA2 mutations? A simulation of comparative cost-effectiveness. [Internet]. Br J Cancer 108:1579-86, 2013Available from: http://www.ncbi.nlm.nih.gov/pubmed/23579217 
15. Griebsch I, Brown J, Boggis C, et al: Cost-effectiveness of screening with contrast enhanced magnetic resonance imaging vs X-ray mammography of women at a high familial risk of breast cancer. Br J Cancer 95:801-810, 2006

16. Moore SG, Shenoy PJ, Fanucchi L, et al: Cost-effectiveness of MRI compared to mammography for breast cancer screening in a high risk population. [Internet]. BMC Health Serv Res 9:9, 2009Available from:

http://www.pubmedcentral.nih.gov/articlerender.fcgi?artid=2630922\&tool=pmcentrez \&rendertype $=$ abstract

17. Pataky R, Armstrong L, Chia S, et al: Cost-effectiveness of MRI for breast cancer screening in BRCA1/2 mutation carriers. [Internet]. BMC Cancer 13:339, 2013Available from:

http://www.pubmedcentral.nih.gov/articlerender.fcgi?artid=3711845\&tool=pmcentrez \&rendertype=abstract

18. Robertson C, Arcot Ragupathy SK, Boachie C, et al: The clinical effectiveness and cost-effectiveness of different surveillance mammography regimens after the treatment for primary breast cancer: systematic reviews registry database analyses and economic evaluation. Health Technol Assess 15, 2011

19. Turnbull LW, Brown SR, Olivier C, et al: Multicentre randomised controlled trial examining the cost-effectiveness of contrast-enhanced high field magnetic resonance imaging in women with primary breast cancer scheduled for wide local excision (COMICE). Health Technol Assess (Rockv) 14:1-158, 2010

20. Onega $T$, Tosteson ANA, Weiss $\mathrm{J}$, et al: Costs of diagnostic and preoperative workup with and without breast MRI in older women with a breast cancer diagnosis. [Internet]. BMC Health Serv Res 16:76, 2016Available from: 
http://bmchealthservres.biomedcentral.com/articles/10.1186/s12913-016-1317-6

21. Mullins CD: Double counting and the reporting of cost per event avoided. Clin Ther 28:602-603, 2006

22. Irvine L, Conroy SP, Sach T, et al: Cost-effectiveness of a day hospital falls prevention programme for screened community-dwelling older people at high risk of falls. Age Ageing 39:710-716, 2010

23. Nshimyumukiza L, Durand A, Gagnon M, et al: An economic evaluation: Simulation of the cost-effectiveness and cost-utility of universal prevention strategies against osteoporosis-related fractures. J Bone Miner Res 28:383-394, 2013 


\section{TABLES}

Table 1. Unit cost data

\section{Resource}

\begin{tabular}{lc}
\hline Most frequent DRGs for breast cancer-related hospital & \\
stays: & \\
Breast-conserving surgery (DRG 09C05J and 09C051) & 2,262 \\
Mastectomy without reconstruction (DRG 09C041) & 3,913 \\
Mastectomy with reconstruction (DRG 09C111) & 8,093 \\
Sentinel lymph node biopsy (DRG 09C101) & 1,180 \\
MRI (including contrast agent) & 302 \\
\hline MRI-guided macrobiopsy & 857 \\
Radiotherapy: & 991 \\
3-dimensional planning & 170 \\
3-dimensional radiation session & 347 \\
2-dimensional planning & 30.3 \\
2-dimensional radiation session & 2.19 \\
\hline Outpatient visits & 30 \\
\hline Daily sick leave compensation & \\
\hline Cost per km per type of transportation & \\
\hline
\end{tabular}

DRG: diagnosis-related group; MRI: magnetic resonance imaging 
Table 2. Healthcare resource use for DCIS treatment over the 6-month period after randomisation

\section{Number of patients}

MRI No MRI
$\mathrm{N}=173$
$\mathrm{N}=172$

\begin{tabular}{lcc}
\hline Hospitalisations related to breast cancer: & & \\
Hospitalisation for initial surgery & $173(100 \%)$ & $172(100 \%)$ \\
Re-hospitalisation & $48(28 \%)$ & $59(34 \%)$ \\
Re-hospitalisation for positive or close & $35(20 \%)$ & $47(27 \%)$ \\
margins & &
\end{tabular}

Type of initial breast surgery received:

- Tumorectomy

160

166

- Mastectomy

16

7

Radiotherapy

$132(76 \%)$

$130(76 \%)$

MRI: magnetic resonance imaging: DCIS: ductal carcinoma in situ 
Table 3. Cost-effectiveness analysis results: MRI versus No MRI

$\begin{array}{ccc}\text { MRI } & \text { No MRI } & \text { Difference [95\%Cl] } \\ n=173 & n=172 & \text { (MRI versus No MRI) } \\ & & \text { Bootstrap } n=10,000\end{array}$

\begin{tabular}{lccc}
\hline Mean cost per patient $(\boldsymbol{\epsilon})$ & 9,980 & 9,682 & $298[-470 ; 1,063]$ \\
$\begin{array}{l}\text { Effectiveness: } \\
\text { Number of re-interventions for }\end{array}$ & 35 & 47 & $-12[-32 ; 4]$ \\
$\begin{array}{l}\text { positive or close margins } \\
\text { Rate of re-intervention }\end{array}$ & $20 \%$ & $27 \%$ & $-7 \%[-17 ; 3]$ \\
\hline
\end{tabular}

MRI: magnetic resonance imaging 
Table 4. Costs per patient over the 6-month period after randomisation

\begin{tabular}{|c|c|c|c|}
\hline Resource & $\begin{array}{c}\text { MRI } \\
\text { Mean } \pm \text { SD } \\
n=173\end{array}$ & $\begin{array}{c}\text { No MRI } \\
\text { Mean } \pm \text { SD } \\
n=172\end{array}$ & $\begin{array}{c}\text { Mean cost } \\
\text { difference }[95 \% \mathrm{Cl}] \\
\text { Bootstrap } \\
n=10,000\end{array}$ \\
\hline $\begin{array}{l}\text { Procedures (including } \\
\text { MRI and biopsies) }\end{array}$ & $545 \pm 325$ & $247 \pm 241$ & $298[237 ; 358]$ \\
\hline $\begin{array}{l}\text { Hospitalisation for initial } \\
\text { surgery }\end{array}$ & $2,458 \pm 587$ & $2,396 \pm 717$ & $62[-79 ; 195]$ \\
\hline $\begin{array}{l}\text { All Re-Hospitalisations } \\
\text { Among which: }\end{array}$ & $922 \pm 1944$ & $1,092 \pm 1,873$ & $-170[-572 ; 81]$ \\
\hline $\begin{array}{l}\text { Re-Hospitalisations } \\
\text { for positive or close } \\
\text { margins }^{1}\end{array}$ & $666 \pm 1,927$ & $931 \pm 1,847$ & $-265[-633 ; 100]$ \\
\hline Radiotherapy & $3,271 \pm 1,940$ & $3,258 \pm 1,941$ & $13[-399 ; 427]$ \\
\hline Sick leaves & $2,647 \pm 2,654$ & $2,554 \pm 3,020$ & $93[-525 ; 683]$ \\
\hline Transportations & $49 \pm 33$ & $51 \pm 29$ & $-2[-8 ; 5]$ \\
\hline Outpatient visits & $88 \pm 68$ & $84 \pm 50$ & $4[-7 ; 18]$ \\
\hline Total cost & $9,980 \pm 3,562$ & $9,682 \pm 3,800$ & $298[-470 ; 1,063]$ \\
\hline
\end{tabular}

MRI: magnetic resonance imaging; SD: standard deviation; $\mathrm{Cl}$ : confidence interval

${ }^{1}$ Cost is weighted by frequency 


\section{Figures}

Figure 1. Cost-effectiveness plane showing the cost difference and difference in effectiveness of MRI versus No MRI; 10,000 bootstrap replications

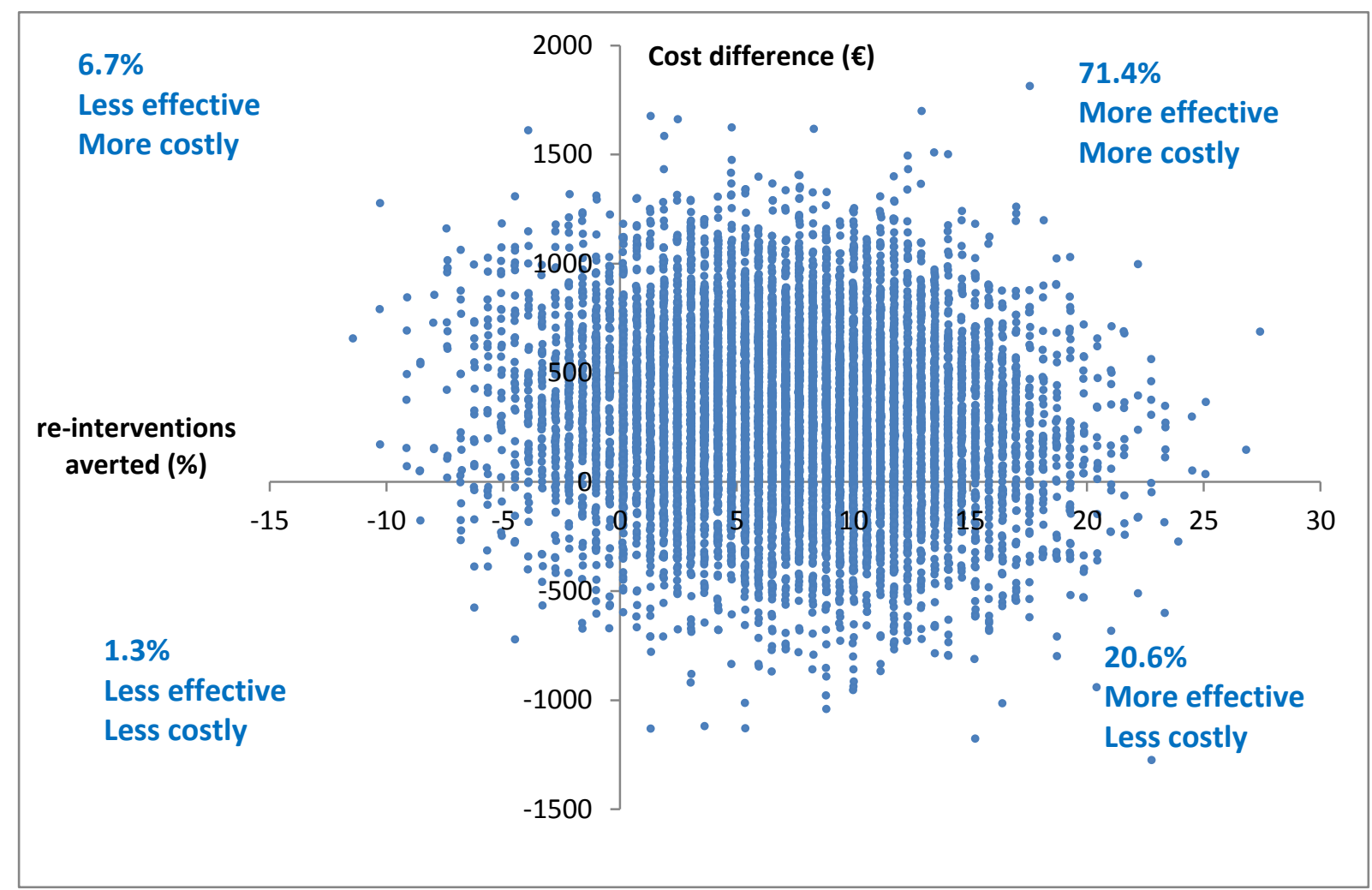


Figure 2. Cost-effectiveness acceptability curve (CEAC) for MRI versus No MRI (10,000 bootstrap replications). The CEAC represents the probability that preoperative MRI is cost-effective compared to standard imaging for various willingness-to-pay thresholds to avert a re-intervention

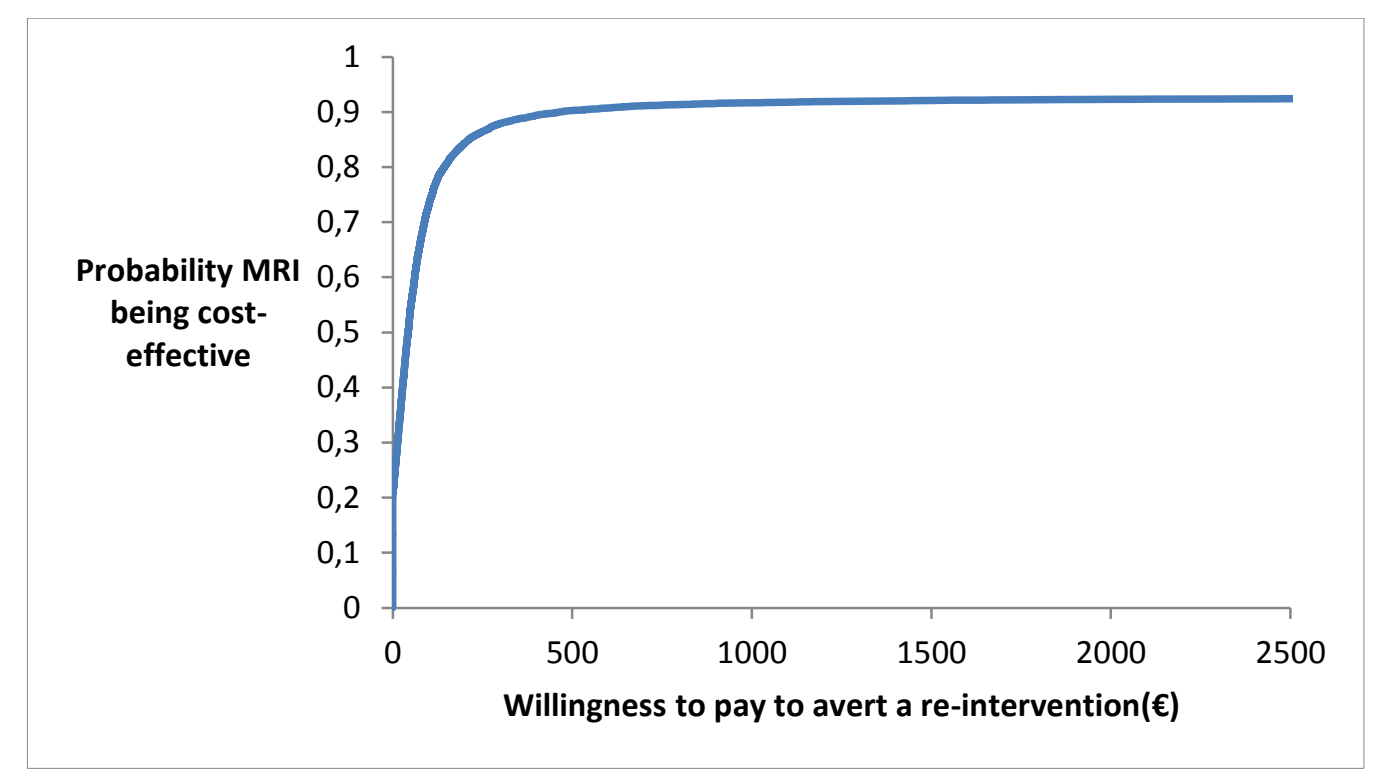

MRI: magnetic resonance imaging 